\title{
WIENER INTEGRAL FOR THE COORDINATE PROCESS UNDER THE $\sigma$-FINITE MEASURE UNIFYING BROWNIAN PENALISATIONS*
}

\author{
KOUJI YANO ${ }^{1}$
}

\begin{abstract}
Wiener integral for the coordinate process is defined under the $\sigma$-finite measure unifying Brownian penalisations, which has been introduced by [Najnudel et al., C. R. Math. Acad. Sci. Paris 345 (2007) 459-466] and [Najnudel et al., MSJ Memoirs 19. Mathematical Society of Japan, Tokyo (2009)]. Its decomposition before and after last exit time from 0 is studied. This study prepares for the author's recent study [K. Yano, J. Funct. Anal. 258 (2010) 3492-3516] of Cameron-Martin formula for the $\sigma$-finite measure.
\end{abstract}

Mathematics Subject Classification. 60H05, 60J65, 46G12.

Received September 29, 2009.

\section{Introduction}

Let $\Omega=C([0, \infty) \rightarrow \mathbb{R})$ and let $\mathcal{F}_{\infty}=\sigma\left(X_{s}: s \geq 0\right)$ where $\left(X_{s}: s \geq 0\right)$ stands for the coordinate process. Let $R^{+}$denote the law on $\left(\Omega, \mathcal{F}_{\infty}\right)$ of the 3 -dimensional Bessel process starting from 0 . We denote by $R^{-}$the law on $\left(\Omega, \mathcal{F}_{\infty}\right)$ of $\left(-X_{t}\right)$ under $R^{+}$. We define $R=\frac{R^{+}+R^{-}}{2}$; in other words, $R$ is the law on $\Omega$ of $\left(\varepsilon X_{t}\right)$ under the product measure $P(\mathrm{~d} \varepsilon) \otimes R^{+}(\mathrm{d} X)$ where $P(\varepsilon=1)=P(\varepsilon=-1)=1 / 2$. For $u>0$ and for two processes $X^{(u)}=\left(X_{t}: 0 \leq t \leq u\right)$ and $Y=\left(Y_{t}: t \geq 0\right)$, we define the concatenation $X^{(u)} \bullet Y$ as

$$
\left(X^{(u)} \bullet Y\right)_{t}= \begin{cases}X_{t} & \text { if } 0 \leq t<u, \\ Y_{t-u} & \text { if } t \geq u \text { and } X_{u}=Y_{0}, \\ X_{u} & \text { if } t \geq u \text { and } X_{u} \neq Y_{0}\end{cases}
$$

We define the concatenation $\Pi^{(u)} \bullet R$ as the law on $\left(\Omega, \mathcal{F}_{\infty}\right)$ of the concatenation $X^{(u)} \bullet Y$ under the product measure $\Pi^{(u)}\left(\mathrm{d} X^{(u)}\right) \otimes R(\mathrm{~d} Y)$.

In this paper we consider the following $\sigma$-finite measure $\mathscr{W}$ defined on $\left(\Omega, \mathcal{F}_{\infty}\right)$ :

$$
\mathscr{W}=\int_{0}^{\infty} \frac{\mathrm{d} u}{\sqrt{2 \pi u}}\left(\Pi^{(u)} \bullet R\right) .
$$

Keywords and phrases. Stochastic integral, Brownian motion, Bessel process, penalisation.

* The research was supported by KAKENHI (20740060).

1 Department of Mathematics, Graduate School of Science, Kobe University, Kobe, Japan. kyano@math.kobe-u.ac.jp 
This measure $\mathscr{W}$ has been introduced by Najnudel et al. [8,9] (see also [13]) in order to give a global view on various Brownian penalisations, which were developed by Roynette et al. (see $[10,11]$ and the references therein).

The purpose of this paper is to define and study Wiener integral, i.e., stochastic integral whose integrand is a deterministic function $f$, written as

$$
\int_{0}^{\infty} f(s) \mathrm{d} X_{s} \text { under } \mathscr{W}
$$

We discuss its decomposition into the sum of two Wiener integrals before and after last exit time from 0; the former is for Brownian bridge, while the latter is for 3-dimensional Bessel process.

As an application of the Wiener integral under $\mathscr{W}$, the author studies, in a separate paper [12], CameronMartin formula for $\mathscr{W}$, i.e., quasi-invariance of $\mathscr{W}$ under deterministic translations.

Our main results are stated in the remainder of this section. All of their proofs will be given in Section 4 .

(1) Wiener integrals. Let us recall definition of Wiener integrals. If the integrand is an indicator, we define

$$
\int_{0}^{\infty} 1_{\left[t_{1}, t_{2}\right)}(s) \mathrm{d} X_{s}=X_{t_{2}}-X_{t_{1}}
$$

We extend it linearly so that we define Wiener integral $\int_{0}^{\infty} f(s) \mathrm{d} X_{s}$ if the integrand $f$ is a step function. In order to extend it to more general integrand functions, we need certain properties peculiar to the process considered; see below.

The following facts are well-known: if a sequence $\left\{f_{n}\right\}$ of step functions approximates $f$ in $\mathrm{L}^{2}(\mathrm{~d} s)$, then, for Brownian motion $\left\{\left(X_{s}\right), W\right\}$, it holds that

$$
\int_{0}^{\infty} f_{n}(s) \mathrm{d} X_{s} \underset{n \rightarrow \infty}{\longrightarrow} \int_{0}^{\infty} f(s) \mathrm{d} X_{s} \quad \text { in } \mathrm{L}^{2}(W)
$$

and, for Brownian bridge $\left\{\left(X_{s}\right), \Pi^{(u)}\right\}$ with $u>0$, it holds that

$$
\int_{0}^{u} f_{n}(s) \mathrm{d} X_{s} \underset{n \rightarrow \infty}{\longrightarrow} \int_{0}^{u} f(s) \mathrm{d} X_{s} \quad \text { in } \mathrm{L}^{2}\left(\Pi^{(u)}\right) .
$$

The symmetrized 3-dimensional Bessel process $\left\{\left(X_{t}\right), R\right\}$ requires an integrand function $f$ to belong to $\mathrm{L}^{2}(\mathrm{~d} s)$ and as well to the following class:

$$
\mathrm{L}^{1}\left(\frac{\mathrm{d} s}{\sqrt{s}}\right)=\left\{f: \int_{0}^{\infty}|f(s)| \frac{\mathrm{d} s}{\sqrt{s}}<\infty\right\} .
$$

Now the following fact holds (see Sect. 3.4 for details): if a sequence $\left\{f_{n}\right\}$ of step functions approximates $f$ both in $\mathrm{L}^{2}(\mathrm{~d} s)$ and in $\mathrm{L}^{1}\left(\frac{\mathrm{d} s}{\sqrt{s}}\right)$, i.e.,

$$
\int_{0}^{\infty}\left|f_{n}(s)-f(s)\right|^{2} \mathrm{~d} s+\int_{0}^{\infty}\left|f_{n}(s)-f(s)\right| \frac{\mathrm{d} s}{\sqrt{s}} \rightarrow 0,
$$

then

$$
\int_{0}^{\infty} f_{n}(s) \mathrm{d} X_{s} \underset{n \rightarrow \infty}{\longrightarrow} \int_{0}^{\infty} f(s) \mathrm{d} X_{s} \quad \text { in } R \text {-probability. }
$$

Note that we cannot dispense with the assumption $f \in \mathrm{L}^{1}\left(\frac{\mathrm{d} s}{\sqrt{s}}\right)$; see Remark 3.13. 
(2) Approximation theorem. Let us discuss the Wiener integral (1.3). Note that the measure $\mathscr{W}_{\text {on }} \mathcal{F}_{\infty}$ is singular to Wiener measure and also to $\operatorname{BES}(3)$ measure; in fact, it holds $\mathscr{W}$-a.e. that $\left|X_{t}\right| \rightarrow \infty$ as $t \rightarrow \infty$ and that $\left(X_{t}\right)$ takes both positive and negative values. Thus, in order to define the Wiener integral (1.3), we can only utilize the definition (1.2).

We need the following notion of convergence. We say that a sequence $\left\{Z_{n}\right\}$ of measurable functionals converge locally in $\mathscr{W}$-measure to a measurable functional $Z$ if, for any $\varepsilon>0$ and any measurable set $A$ with $\mathscr{W}(A)<\infty$,

$$
\mathscr{W}\left(A \cap\left\{\left|Z_{n}-Z\right| \geq \varepsilon\right\}\right) \rightarrow 0 \quad \text { as } n \rightarrow \infty
$$

Thanks to the $\sigma$-finiteness of $\mathscr{W}$, this notion of convergence plays a key role; see Section 2 for details, and also $[1]$.

We introduce the following class of functions:

$$
\mathrm{L}^{1}\left(\frac{\mathrm{d} s}{1+\sqrt{s}}\right)=\left\{f: \int_{0}^{\infty}|f(s)| \frac{\mathrm{d} s}{1+\sqrt{s}}<\infty\right\}
$$

Note that $f \in \mathrm{L}^{1}\left(\frac{\mathrm{d} s}{1+\sqrt{s}}\right)$ if and only if $f$ is locally integrable and $\int_{v}^{\infty}|f(s)| \frac{\mathrm{d} s}{\sqrt{s}}<\infty$ for any (large) $v>0$. Wiener integral for $X$ under $\mathscr{W}$ may be defined through the following theorem.

Theorem 1.1. Let $f \in \mathrm{L}^{2}(\mathrm{~d} s) \cap \mathrm{L}^{1}\left(\frac{\mathrm{d} s}{1+\sqrt{s}}\right)$. Let $\left\{f_{n}\right\}$ be a sequence of step functions such that $\left\{f_{n}\right\}$ approximates $f$ both in $\mathrm{L}^{2}(\mathrm{~d} s)$ and in $\mathrm{L}^{1}\left(\frac{\mathrm{d} s}{1+\sqrt{s}}\right)$, i.e.,

$$
\int_{0}^{\infty}\left|f_{n}(s)-f(s)\right|^{2} \mathrm{~d} s+\int_{0}^{\infty}\left|f_{n}(s)-f(s)\right| \frac{\mathrm{d} s}{1+\sqrt{s}} \rightarrow 0
$$

(Note that this condition is weaker than (1.8).) Then there exists a random variable, which will be denoted by $\int_{0}^{\infty} f(s) \mathrm{d} X_{s}$, such that

$$
\int_{0}^{\infty} f_{n}(s) \mathrm{d} X_{s} \underset{n \rightarrow \infty}{\longrightarrow} \int_{0}^{\infty} f(s) \mathrm{d} X_{s} \quad \text { locally in } \mathscr{W} \text {-measure. }
$$

The limit random variable does not depend up to $\mathscr{W}$-null sets on the choice of the approximation sequence $\left\{f_{n}\right\}$.

Since $\mathrm{L}^{1}(\mathrm{~d} s) \subset \mathrm{L}^{1}\left(\frac{\mathrm{d} s}{1+\sqrt{s}}\right)$, the following corollary is immediate from Theorem 1.1.

Corollary 1.2. Let $f \in \mathrm{L}^{2}(\mathrm{~d} s) \cap \mathrm{L}^{1}(\mathrm{~d} s)$. Let $\left\{f_{n}\right\}$ be a sequence of step functions such that $\left\{f_{n}\right\}$ approximates $f$ both in $\mathrm{L}^{2}(\mathrm{~d} s)$ and in $\mathrm{L}^{1}(\mathrm{~d} s)$. Then it holds that

$$
\int_{0}^{\infty} f_{n}(s) \mathrm{d} X_{s} \underset{n \rightarrow \infty}{\longrightarrow} \int_{0}^{\infty} f(s) \mathrm{d} X_{s} \quad \text { locally in } \mathscr{W} \text {-measure. }
$$


(3) Decomposition before and after last exit time. Let $g(X)$ denote the last exit time from 0 for $X$ :

$$
g(X)=\sup \left\{s \geq 0: X_{s}=0\right\} .
$$

For $u \geq 0$, let $\theta_{u} X$ denote the shifted process: $\left(\theta_{u} X\right)_{s}=X_{u+s}, s \geq 0$. Then the definition (1.2) says that the measure $\mathscr{W}$ may be described as follows:

(i) $\mathscr{W}(g(X) \in \mathrm{d} u)=\frac{\mathrm{d} u}{\sqrt{2 \pi u}}$;

(ii) For (Lebesgue) a.e. $u \in[0, \infty)$, it holds that, given $g(X)=u$,

(iia) $\left(X_{s}: s \leq u\right)$ is a Brownian bridge from 0 to 0 of length $u$;

(iib) $\left(\left(\theta_{u} X\right)_{s}: s \geq 0\right)$ is a symmetrized 3-dimensional Bessel process.

Based on this path decomposition, we may also decompose the Wiener integral as follows.

Theorem 1.3. Let $f \in \mathrm{L}^{2}(\mathrm{~d} s) \cap \mathrm{L}^{1}\left(\frac{\mathrm{d} s}{1+\sqrt{s}}\right)$. Then it holds that

$$
f(\cdot+u) \in \mathrm{L}^{1}\left(\frac{\mathrm{d} s}{\sqrt{s}}\right) \quad \text { for a.e. } u \in[0, \infty)
$$

and that there exists a jointly measurable functional $(u, X) \mapsto I(f ; u, X)$ such that

$$
\int_{0}^{\infty} f(s) \mathrm{d} X_{s}=I(f ; g(X), X) \quad \mathscr{W} \text {-a.e. }
$$

and that, for a.e. $u \in[0, \infty)$,

$$
I(f ; u, X)=\int_{0}^{u} f(s) \mathrm{d} X_{s}+\int_{0}^{\infty} f(s+u) \mathrm{d}\left(\theta_{u} X\right)_{s} \quad\left(\Pi^{(u)} \bullet R\right) \text {-a.e. }
$$

In the right hand side of the expression (1.18), the first and the second terms are Wiener integrals for the Brownian bridge $\left(X_{s}: s \leq u\right)$ and for the symmetrized 3-dimensional Bessel process $\left(\left(\theta_{u} X\right)_{s}: s \geq 0\right)$, respectively.

(4) Continuous modification. Let $0<T<\infty$ be fixed and write $\mathcal{F}_{T}=\sigma\left(X_{s}: s \in[0, T]\right)$. Note that an $\mathcal{F}_{T}$-measurable set is $\mathscr{W}$-null if and only if it is $W$-null. Although we cannot apply Radon-Nikodym theorem to $\mathscr{W}$ since it is not $\sigma$-finite on $\mathcal{F}_{T}$, we have the following absolute continuity relationship (see [9], Eq. (1.2.45)):

$$
\mathscr{W}\left[F_{T}(X) \mathrm{e}^{-g(X)}\right]=W\left[F_{T}(X) \Lambda_{T}(X)\right]
$$

for any bounded $\mathcal{F}_{T}$-measurable functional $F_{T}(X)$, where $\Lambda_{T}(X)$ is given as

$$
\Lambda_{T}(X)=\left|X_{T}\right| \exp \left(-g^{(T)}(X)\right)+\int_{0}^{\infty} \frac{\mathrm{d} u}{\sqrt{2 \pi u}} \mathrm{e}^{-(T+u)} \exp \left(-\frac{X_{T}^{2}}{2 u}\right)
$$

and where $g^{(T)}(X)=\sup \left\{s \leq T: X_{s}=0\right\}$. This shows that, if we assume that $f \in \mathrm{L}^{2}([0, T]$, ds), there exists, under $\mathscr{W}$, a continuous modification $\left\{\int_{0}^{t} f(s) \mathrm{d} X_{s}: t \in[0, T]\right\}$ of $\left\{\int_{0}^{\infty} 1_{[0, t)} f(s) \mathrm{d} X_{s}: t \in[0, T]\right\}$. It is not, however, immediate from this fact whether there exists a jointly measurable functional which gives the decomposition of the continuous modification before and after the last exit time. The following theorem assures existence of such a functional.

Theorem 1.4. Let $f \in \mathrm{L}^{2}([0, T], \mathrm{d} s)$. Then there exists a jointly measurable functional $(t, u, X) \mapsto I_{t}(f ; u, X)$ such that the following statements hold:

(i) For a.e. $u \in[0, \infty)$ and for $\left(\Pi^{(u)} \bullet R\right)(\mathrm{d} X)$-a.e. $X$, the function $t \mapsto I_{t}(f ; u, X)$ is continuous; 
(ii) For each $t \in[0, T]$,

$$
\int_{0}^{t} f(s) \mathrm{d} X_{s}=I_{t}(f ; g(X), X) \quad \mathscr{W} \text {-a.e.; }
$$

(iii) For each $t \in[0, T]$ and for a.e. $u \in[0, \infty)$,

$$
I_{t}(f ; u, X)=\int_{0}^{u \wedge t} f(s) \mathrm{d} X_{s}+\int_{0}^{(t-u) \vee 0} f(s+u) \mathrm{d}\left(\theta_{u} X\right)_{s} \quad\left(\Pi^{(u)} \bullet R\right) \text {-a.e. }
$$

(5) This paper is organized as follows. In Section 2, we study several properties of convergence a.e. and of convergence locally in measure, both considered on a $\sigma$-finite measure space. In Section 3 , we recall Wiener integrals for Brownian motion, Brownian bridge and 3-dimensional Bessel process. Section 4 is devoted to the proofs of the main theorems.

\section{Convergence locally in $\mathscr{W}$-measure}

In this subsection, we only assume that $(\Omega, \mathcal{F}, \mathscr{W})$ is a $\sigma$-finite measure space.

Definition 2.1. Let $Z, Z_{1}, Z_{2}, \ldots$ be $\mathcal{F}$-measurable functionals. As $n \rightarrow \infty$, we say that $Z_{n} \rightarrow Z$ locally in $\mathscr{W}$-measure if, for any $\varepsilon>0$ and any $A \in \mathcal{F}$ with $\mathscr{W}(A)<\infty$, it holds that

$$
\mathscr{W}\left(A \cap\left\{\left|Z_{n}-Z\right| \geq \varepsilon\right\}\right) \rightarrow 0 .
$$

Let us study some properties about this convergence. Define

$$
\mathrm{L}_{+}^{1}(\mathscr{W})=\left\{G: \Omega \rightarrow \mathbb{R}_{+}, \mathcal{F} \text {-measurable, } \mathscr{W}(G=0)=0, \mathscr{W}[G]<\infty\right\} .
$$

For $G \in \mathrm{L}_{+}^{1}(\mathscr{W})$, we define a probability measure $\mathscr{W}^{G}$ on $(\Omega, \mathcal{F})$ by

$$
\mathscr{W}^{G}(A)=\frac{\mathscr{W}\left[1_{A} G\right]}{\mathscr{W}[G]}, \quad A \in \mathcal{F}
$$

We obtain the following lemma, which only requires the $\sigma$-finiteness of $\mathscr{W}$.

Proposition 2.2. Let $Z, Z_{1}, Z_{2}, \ldots$ be $\mathcal{F}$-measurable functionals.

(i) The following three statements are equivalent:

(A1) $Z_{n} \rightarrow Z \mathscr{W}$-a.e.

(A2) $Z_{n} \rightarrow Z \mathscr{W}^{G}$-a.s. for some $G \in \mathrm{L}_{+}^{1}(\mathscr{W})$.

(A3) $Z_{n} \rightarrow Z \mathscr{W}^{G}$-a.s. for any $G \in \mathrm{L}_{+}^{1}(\mathscr{W})$.

(ii) The following three statements are equivalent:

(B1) $Z_{n} \rightarrow Z$ locally in $\mathscr{W}$-measure.

(B2) $Z_{n} \rightarrow Z$ in $\mathscr{W}^{G}$-probability for some $G \in \mathrm{L}_{+}^{1}(\mathscr{W})$.

(B3) $Z_{n} \rightarrow Z$ in $\mathscr{W}^{G}$-probability for any $G \in \mathrm{L}_{+}^{1}(\mathscr{W})$.

(iii) $Z_{n} \rightarrow Z$ locally in $\mathscr{W}$-measure if and only if one can extract, from an arbitrary subsequence, a further subsequence $\{n(k): k=1,2, \ldots\}$ along which $Z_{n(k)} \rightarrow Z \mathscr{W}$-a.e.

(iv) Convergence locally in $\mathscr{W}$-measure may be induced by some complete separable metric on the set of $\mathcal{F}_{\infty}$-measurable functionals.

The reader may not be familiar with claim (ii), so we give its proof for convenience of the reader, although it is an elementary argument. 
Proof of claim (ii) of Proposition 2.2. Note that, since $\mathscr{W}$ is $\sigma$-finite, we may take a family $\left\{E_{m}\right\} \subset \mathcal{F}$ such that $0<\mathscr{W}\left(E_{m}\right)<\infty$ and $\cup_{m} E_{m}=\Omega$.

$[(\mathrm{B} 3) \Rightarrow(\mathrm{B} 1)]$ Suppose that $Z_{m} \rightarrow Z$ in $\mathscr{W}^{G}$-probability for any $G \in \mathrm{L}_{+}^{1}(\mathscr{W})$. Let $A \in \mathcal{F}$ such that $\mathscr{W}(A)<\infty$. We define $G=1_{A}+\sum_{m=1}^{\infty} 2^{-m \mathscr{W}}\left(E_{m}\right)^{-1} 1_{E_{m}}$. Then we have $G \in \mathrm{L}_{+}^{1}(\mathscr{W})$, and consequently, we obtain $Z_{n} \rightarrow Z$ in $\mathscr{W}^{G}$-probability. For any $\varepsilon>0$, we have

$$
\mathscr{W}\left(A \cap\left\{\left|Z_{n}-Z\right| \geq \varepsilon\right\}\right) \leq \mathscr{W}[G] \mathscr{W}^{G}\left(\left|Z_{n}-Z\right| \geq \varepsilon\right) \rightarrow 0 .
$$

Hence we obtain (B1).

$[(\mathrm{B} 1) \Rightarrow(\mathrm{B} 2)]$ Suppose that $Z_{n} \rightarrow Z$ locally in $\mathscr{W}$-measure. Then, for any $\varepsilon>0$, we have

$$
\sum_{m=1}^{\infty} \frac{1}{2^{m} \mathscr{W}\left(E_{m}\right)} \mathscr{W}\left(E_{m} \cap\left\{\left|Z_{n}-Z\right| \geq \varepsilon\right\}\right) \underset{n \rightarrow \infty}{\longrightarrow} 0 .
$$

Now we define $G=\sum_{m=1}^{\infty} 2^{-m} \mathscr{W}\left(E_{m}\right)^{-1} 1_{E_{m}}$. Then we have $G \in \mathrm{L}_{+}^{1}(\mathscr{W})$ and obtain $Z_{n} \rightarrow Z$ in $\mathscr{W}^{G}$-probability.

[(B2) $\Rightarrow(\mathrm{B} 3)]$ Suppose that $Z_{n} \rightarrow Z$ in $\mathscr{W}^{G}$-probability for $G \in \mathrm{L}_{+}^{1}(\mathscr{W})$. Then, from any subsequence $k(n) \rightarrow \infty$, we can extract a further subsequence $k^{\prime}(n) \rightarrow \infty$ along which $Z_{k^{\prime}(n)} \rightarrow Z, \mathscr{W}^{G}$-a.s., and consequently by (i), $Z_{k^{\prime}(n)} \rightarrow Z, \mathscr{W}$-a.e. Hence, again by (i), we obtain (B3). The proof of claim (ii) is now complete.

\section{Wiener integrals for Brownian motion, Brownian Bridge and 3-Dimensional BESSEL PROCESS}

\subsection{Wiener integrals}

Let $\Omega=C([0, \infty) \rightarrow \mathbb{R})$ and $X=\left(X_{t}: t \geq 0\right)$ stand for the coordinate process. For $0<u<\infty$, let $\mathcal{S}([0, u])$ denote the set of all step functions $f$ on $[0, u]$ of the form:

$$
f(t)=\sum_{k=1}^{n} c_{k} 1_{\left[t_{k-1}, t_{k}\right)}(t), \quad t \geq 0
$$

with $n \in \mathbb{N}, c_{k} \in \mathbb{R}(k=1, \ldots, n)$ and $0=t_{0}<t_{1}<\cdots<t_{n}<u$. Note that $\mathcal{S}([0, u])$ is dense in $\mathrm{L}^{2}([0, u], \mathrm{d} s)$; indeed, we can take two sequences $\left\{f_{n}^{+}\right\}$and $\left\{f_{n}^{-}\right\}$from $\mathcal{S}([0, u])$ such that $0 \leq f_{n}^{+} \nearrow f \vee 0$ and $0 \leq f_{n}^{-} \nearrow(-f) \vee 0$, and hence we see that $f_{n}^{+}-f_{n}^{-} \rightarrow f$ in $\mathrm{L}^{2}([0, u], \mathrm{d} s)$. We write

$$
\mathcal{S}=\left\{f: \exists u \in[0, \infty) \text { such that }\left.f\right|_{[u, \infty)}=0 \text { and }\left.f\right|_{[0, u)} \in \mathcal{S}([0, u])\right\} .
$$

Then we know that $\mathcal{S}$ is dense in $\mathrm{L}^{2}(\mathrm{~d} s)$.

For a function $f \in \mathcal{S}$ and a process $X$, we define

$$
\int_{0}^{\infty} f(s) \mathrm{d} X_{s}=\sum_{k=1}^{n} c_{k}\left(X_{t_{k}}-X_{t_{k-1}}\right) .
$$

For a more general function $f$, we will write $\int_{0}^{\infty} f(s) \mathrm{d} X_{s}$ for the limit of $\int_{0}^{\infty} f_{n}(s) \mathrm{d} X_{s}$ in some sense with an approximation sequence $\left\{f_{n}\right\} \subset \mathcal{S}$ of $f$ in some functional space, and call it Wiener integral of $f$ for $X$ whenever it is well-defined.

In the following subsections, we give an introductory review on how to construct Wiener integrals for Brownian motion, Brownian bridge and 3-dimensional Bessel process, and on several facts about them. 


\subsection{Wiener integral for Brownian motion}

Denote $\mathcal{F}_{\infty}=\sigma\left(X_{s}: s \geq 0\right)$. Let $W$ denote the Wiener measure, i.e., the law on $\left(\Omega, \mathcal{F}_{\infty}\right)$ of a (onedimensional, standard) Brownian motion. Let us recall Wiener integral for the Brownian motion $\left\{\left(X_{t}\right), W\right\}$.

The key to approximation is the following identity:

Theorem 3.1 (Itô isometry). For any $f \in \mathcal{S}$, it holds that

$$
W\left[\left|\int_{0}^{\infty} f(s) \mathrm{d} X_{s}\right|^{2}\right]=\int_{0}^{\infty}|f(s)|^{2} \mathrm{~d} s .
$$

Although it is widely known, we give the proof for completeness of the paper.

Proof. Let $f \in \mathcal{S}$ be of the form (3.1). Since $\left\{X_{t_{k}}-X_{t_{k-1}}: k=1, \ldots, n\right\}$ is an orthogonal system in $\mathrm{L}^{2}(W)$ and since $W\left[\left(X_{t_{k}}-X_{t_{k-1}}\right)^{2}\right]=t_{k}-t_{k-1}$, we have

$$
W\left[\left|\sum_{k=1}^{n} c_{k}\left(X_{t_{k}}-X_{t_{k-1}}\right)\right|^{2}\right]=\sum_{k=1}^{n} c_{k}^{2}\left(t_{k}-t_{k-1}\right) .
$$

This proves (3.4).

The Itô isometry (3.4) shows that if $\left\{f_{n}\right\} \subset \mathcal{S}$ approximates $f$ in $\mathrm{L}^{2}(\mathrm{~d} s)$, then the Wiener integral $\int_{0}^{\infty} f_{n}(s) \mathrm{d} X_{s}$ forms a Cauchy sequence in $\mathrm{L}^{2}(W)$ and hence it converges in $\mathrm{L}^{2}(W)$ where the limit random variable does not depend on the choice of the approximation sequence $\left\{f_{n}\right\}$.

Definition 3.2. For $f \in \mathrm{L}^{2}(\mathrm{~d} s)$, the Wiener integral $\int_{0}^{\infty} f(s) \mathrm{d} X_{s}$ is defined as the $\mathrm{L}^{2}(W)$-limit of $\int_{0}^{\infty} f_{n}(s) \mathrm{d} X_{s}$ for some sequence $\left\{f_{n}\right\} \subset \mathcal{S}$ which approximates $f$ in $\mathrm{L}^{2}(\mathrm{~d} s)$.

Theorem 3.3. For any $f \in \mathrm{L}^{2}(\mathrm{~d} s)$, the Wiener integral $\int_{0}^{\infty} f(s) \mathrm{d} X_{s}$ satisfies the Itô isometry (3.4) and is a centered Gaussian variable with variance $\|f\|_{\mathrm{L}^{2}(\mathrm{~d} s)}^{2}$.

Proof. The former assertion is immediate from Theorem 3.1. The latter is obvious via characteristic functions.

\subsection{Wiener integral for Brownian bridge}

Let $0<u<\infty$ be fixed. We write $\Omega^{(u)}=C([0, u] \rightarrow \mathbb{R})$ and write $X^{(u)}=\left(X_{s}: 0 \leq s \leq u\right)$ for the coordinate process. (We sometimes use the same symbol $X^{(u)}$ to mean the part $\left(X_{s}: 0 \leq s \leq u\right)$ of the coordinate process $\left(X_{s}: s \geq 0\right)$ of $\Omega=C([0, \infty) \rightarrow \mathbb{R})$.) Denote $\mathcal{F}_{u}=\sigma\left(X_{s}: s \leq u\right)$. We denote by $\Pi^{(u)}$ the law on $\left(\Omega^{(u)}, \mathcal{F}_{u}\right)$ of the Brownian bridge:

$$
\Pi^{(u)}(\cdot)=W\left(\cdot \mid X_{u}=0\right) .
$$

The process $X^{(u)}$ under $\Pi^{(u)}$ is a continuous centered Gaussian process with covariance $\Pi^{(u)}\left[X_{s} X_{t}\right]=s-s t / u$ for $0 \leq s \leq t \leq u$. As a realization of $\left\{\left(X_{s}\right), \Pi^{(u)}\right\}$, we may take

$$
\left(B_{s}-\frac{s}{u} B_{u}: s \in[0, u]\right)
$$

where $\left\{\left(B_{t}\right), \Pi^{(u)}\right\}$ is a Brownian motion. 
Let us recall Wiener integral for Brownian bridge $\left\{X^{(u)}, \Pi^{(u)}\right\}$ for $0<u<\infty$. (See also [6].) For $f \in$ $\mathrm{L}^{2}([0, u], \mathrm{d} s)$, we define $\pi_{u} f \in \mathrm{L}^{2}([0, u], \mathrm{d} s)$ by

$$
\left(\pi_{u} f\right)(s)=f(s)-\frac{1}{u} \int_{0}^{u} f(t) \mathrm{d} t, \quad s \in[0, u] .
$$

Note that $\int_{0}^{u}\left(\pi_{u} f\right)(s) \mathrm{d} s=0$ and that

$$
\left\|\pi_{u} f\right\|_{\mathrm{L}^{2}([0, u], \mathrm{d} s)}^{2}=\|f\|_{\mathrm{L}^{2}([0, u], \mathrm{d} s)}^{2}-\frac{1}{u}\left(\int_{0}^{u} f(t) \mathrm{d} t\right)^{2} .
$$

In particular, we have

$$
\left\|\pi_{u} f\right\|_{\mathrm{L}^{2}([0, u], \mathrm{d} s)} \leq\|f\|_{\mathrm{L}^{2}([0, u], \mathrm{d} s)} .
$$

Theorem 3.4 (Itô isometry for Brownian bridge). For any $f \in \mathcal{S}([0, u])$, it holds that

$$
\Pi^{(u)}\left[\left(\int_{0}^{u} f(s) \mathrm{d} X_{s}\right)^{2}\right]=\int_{0}^{u}\left|\pi_{u} f(s)\right|^{2} \mathrm{~d} s .
$$

Although it is elementary, we give the proof for completeness of the paper.

Proof. Let us adopt the realization (3.7) of the Brownian bridge. Taking the Wiener integrals for $\left(B_{t}\right)$ of both sides of (3.8), we obtain

$$
\int_{0}^{u} f(s) \mathrm{d} B_{s}-\frac{B_{u}}{u} \int_{0}^{u} f(s) \mathrm{d} s=\int_{0}^{u}\left(\pi_{u} f\right)(s) \mathrm{d} B_{s} .
$$

This shows that

$$
\int_{0}^{u} f(s) \mathrm{d} X_{s}=\int_{0}^{u}\left(\pi_{u} f\right)(s) \mathrm{d} B_{s}
$$

for all $f \in \mathcal{S}([0, u])$. Thus we obtain (3.11) from the Itô isometry (3.4) for Wiener integral for $\left(B_{t}\right)$.

The Itô isometry (3.11) and inequality (3.10) show that if $\left\{f_{n}\right\} \subset \mathcal{S}([0, u])$ approximates $f$ in $\mathrm{L}^{2}([0, u], \mathrm{d} s)$, then the Wiener integral $\int_{0}^{\infty} f_{n}(s) \mathrm{d} X_{s}$ forms a Cauchy sequence in $\mathrm{L}^{2}\left(\Pi^{(u)}\right)$ and hence it converges in $\mathrm{L}^{2}\left(\Pi^{(u)}\right)$ where the limit random variable does not depend on the choice of the approximation sequence $\left\{f_{n}\right\}$.

Definition 3.5. For $f \in \mathrm{L}^{2}([0, u], \mathrm{d} s)$, the Wiener integral $\int_{0}^{\infty} f(s) \mathrm{d} X_{s}$ is defined as the $\mathrm{L}^{2}\left(\Pi^{(u)}\right)$-limit of $\int_{0}^{\infty} f_{n}(s) \mathrm{d} X_{s}$ for some sequence $\left\{f_{n}\right\} \subset \mathcal{S}$ which approximates $f$ in $\mathrm{L}^{2}([0, u], \mathrm{d} s)$.

Theorem 3.6. For any $f \in \mathrm{L}^{2}([0, u], \mathrm{d} s)$, the Wiener integral $\int_{0}^{\infty} f(s) \mathrm{d} X_{s}$ satisfies the Itô isometry (3.11) and identity (3.13), and is a centered Gaussian variable with variance $\left\|\pi_{u} f\right\|_{\mathrm{L}^{2}([0, u], \mathrm{d} s)}^{2}$.

Proof. The former assertion is immediate from Theorem 3.4. The latter is obvious via characteristic functions.

\subsection{Wiener integral for 3-dimensional Bessel process via stochastic differential equation}

Recall that $R^{+}$is the law on $\left(\Omega, \mathcal{F}_{\infty}\right)$ of the 3 -dimensional Bessel process starting from 0 . It is well-known that $R^{+}$is the law of the process $\left(\sqrt{Z_{t}}\right)$ where $\left(Z_{t}\right)$ is the unique strong solution to the stochastic differential equation

$$
\mathrm{d} Z_{t}=2 \sqrt{\left|Z_{t}\right|} \mathrm{d} \beta_{t}+3 \mathrm{~d} t, \quad Z_{0}=0
$$


with $\left(\beta_{t}\right)$ a Brownian motion. Under $R^{+}$, the process $X$ satisfies

$$
\mathrm{d} X_{t}=\mathrm{d} B_{t}+\frac{1}{X_{t}} \mathrm{~d} t, \quad X_{0}=0
$$

with a Brownian motion $\left\{\left(B_{t}\right), R^{+}\right\}$.

We may define Wiener integral for 3-dimensional Bessel process $\left\{\left(X_{t}\right), R^{+}\right\}$via the stochastic differential equation (3.15). Noting that

$$
R^{+}\left[\frac{1}{X_{t}}\right]=\sqrt{\frac{2}{\pi t}}
$$

we may give the following definition.

Definition 3.7. For $f \in \mathrm{L}^{2}(\mathrm{~d} s) \cap \mathrm{L}^{1}\left(\frac{\mathrm{d} s}{\sqrt{s}}\right)$, we define

$$
\int_{0}^{\infty} f(s) \mathrm{d} X_{s}=\int_{0}^{\infty} f(s) \mathrm{d} B_{s}+\int_{0}^{\infty} \frac{f(s)}{X_{s}} \mathrm{~d} s .
$$

Approximation by step functions is given as follows.

Lemma 3.8. Let $f \in \mathrm{L}^{2}(\mathrm{~d} s) \cap \mathrm{L}^{1}\left(\frac{\mathrm{d} s}{\sqrt{s}}\right)$. Suppose that a sequence $\left\{f_{n}\right\} \subset \mathcal{S}$ approximates $f$ both in $\mathrm{L}^{2}(\mathrm{~d} s)$ and in $\mathrm{L}^{1}\left(\frac{\mathrm{d} s}{\sqrt{s}}\right)$ (see (1.8)). Then it holds that

$$
\int_{0}^{\infty} f_{n}(s) \mathrm{d} X_{s} \underset{n \rightarrow \infty}{\longrightarrow} \int_{0}^{\infty} f(s) \mathrm{d} X_{s} \quad \text { in } \mathrm{L}^{1}\left(R^{+}\right) .
$$

Proof. Since $f_{n} \rightarrow f$ in $\mathrm{L}^{2}(\mathrm{~d} s)$, we have

$$
\int_{0}^{\infty} f_{n}(s) \mathrm{d} B_{s} \underset{n \rightarrow \infty}{\longrightarrow} \int_{0}^{\infty} f(s) \mathrm{d} B_{s} \quad \text { in } \mathrm{L}^{2}\left(R^{+}\right),
$$

and consequently, the convergence occurs also in $\mathrm{L}^{1}\left(R^{+}\right)$. Since

$$
R^{+}\left[\int_{0}^{\infty} \frac{\left|f_{n}(s)-f(s)\right|}{X_{s}} \mathrm{~d} s\right]=\sqrt{\frac{2}{\pi}} \int_{0}^{\infty}\left|f_{n}(s)-f(s)\right| \frac{\mathrm{d} s}{\sqrt{s}} \underset{n \rightarrow \infty}{\longrightarrow} 0
$$

we have

$$
\int_{0}^{\infty} \frac{f_{n}(s)}{X_{s}} \mathrm{~d} s \underset{n \rightarrow \infty}{\longrightarrow} \int_{0}^{\infty} \frac{f(s)}{X_{s}} \mathrm{~d} s \quad \text { in } \mathrm{L}^{1}\left(R^{+}\right)
$$

The proof is now complete.

\subsection{Wiener integral for 3-dimensional Bessel process via centered Bessel process}

There is another way of constructing Wiener integral for the 3-dimensional Bessel process, which is due to Funaki et al. [2] (see also [3-5]). Note that this method will play a crucial role in [12].

We define

$$
\widehat{X}_{t}=X_{t}-R^{+}\left[X_{t}\right]
$$


and we call $\left\{\left(\widehat{X}_{t}\right), R^{+}\right\}$the centered 3-dimensional Bessel process. For $f \in \mathcal{S}$ of the form (3.1), the Wiener integral has already been defined as

$$
\int_{0}^{\infty} f(s) \mathrm{d} \widehat{X}_{s}=\sum_{k=1}^{n} c_{k}\left(\widehat{X}_{t_{k}}-\widehat{X}_{t_{k-1}}\right) .
$$

We remark that neither the 3-dimensional Bessel process nor the centered one is Gaussian. So we cannot expect an isometry to hold similar to the Itô isometries (3.4) and (3.11). Funaki et al. [2] obtained the following remarkable inequality analogous to the Itô isometries.

Theorem 3.9 (Funaki et al. [2]). For any $f \in \mathcal{S}$ and any non-negative convex function $\psi$ on $\mathbb{R}$, it holds that

$$
R^{+}\left[\psi\left(\int_{0}^{\infty} f(s) \mathrm{d} \widehat{X}_{s}\right)\right] \leq W\left[\psi\left(\int_{0}^{\infty} f(s) \mathrm{d} X_{s}\right)\right] .
$$

In particular, taking $\psi(x)=x^{2}$, one has

$$
R^{+}\left[\left|\int_{0}^{\infty} f(s) \mathrm{d} \widehat{X}_{s}\right|^{2}\right] \leq \int_{0}^{\infty}|f(s)|^{2} \mathrm{~d} s .
$$

For the proof of this theorem, see [2], Proposition 4.1.

The inequality (3.25) shows that, if $\left\{f_{n}\right\} \subset \mathcal{S}$ approximates $f$ in $\mathrm{L}^{2}(\mathrm{~d} s)$, then the Wiener integral $\int_{0}^{\infty} f_{n}(s) \mathrm{d} \widehat{X}_{s}$ forms a Cauchy sequence in $\mathrm{L}^{2}\left(R^{+}\right)$and hence it converges in $\mathrm{L}^{2}\left(R^{+}\right)$where the limit random variable does not depend on the choice of the approximation sequence $\left\{f_{n}\right\}$.

Definition 3.10. For $f \in \mathrm{L}^{2}(\mathrm{~d} s)$, the Wiener integral $\int_{0}^{\infty} f(s) \mathrm{d} \widehat{X}_{s}$ is defined as the $\mathrm{L}^{2}\left(R^{+}\right)$-limit of $\int_{0}^{\infty} f_{n}(s) \mathrm{d} \widehat{X}_{s}$ for some sequence $\left\{f_{n}\right\} \subset \mathcal{S}$ which approximates $f$ in $\mathrm{L}^{2}(\mathrm{~d} s)$.

Theorem 3.11. For any $f \in \mathrm{L}^{2}(\mathrm{~d} s)$ and any non-negative convex function $\psi$ on $\mathbb{R}$, the inequality (3.24) remains valid, and so does (3.25), in particular.

Proof. We may take an approximation sequence $\left\{f_{n}\right\}$ of $f$ in $\mathrm{L}^{2}(\mathrm{~d} s)$ so that $\sigma_{n}:=\left\|f_{n}\right\|_{\mathrm{L}^{2}(\mathrm{~d} s)}$ converges increasingly to $\sigma:=\|f\|_{\mathrm{L}^{2}(\mathrm{~d} s)}$. By the monotone convergence theorem, we see that

$$
\int_{-\infty}^{\infty} \psi(x) \exp \left(-\frac{x^{2}}{2 \sigma_{n}}\right) \mathrm{d} x \underset{n \rightarrow \infty}{\longrightarrow} \int_{-\infty}^{\infty} \psi(x) \exp \left(-\frac{x^{2}}{2 \sigma}\right) \mathrm{d} x
$$

(The limit may possibly be infinite.) Since the Wiener integral for Brownian motion is Gaussian, we obtain

$$
W\left[\psi\left(\int_{0}^{\infty} f_{n}(s) \mathrm{d} X_{s}\right)\right] \underset{n \rightarrow \infty}{\longrightarrow} W\left[\psi\left(\int_{0}^{\infty} f(s) \mathrm{d} X_{s}\right)\right] .
$$

Therefore we obtain (3.24) by Fatou's lemma and by Theorem 3.9.

Note that

$$
R^{+}\left[X_{t}\right]=\sqrt{\frac{2}{\pi}} \int_{0}^{t} \frac{\mathrm{d} s}{\sqrt{s}}, \quad t \geq 0 .
$$

Lemma 3.12. Let $f \in \mathrm{L}^{2}(\mathrm{~d} s) \cap \mathrm{L}^{1}\left(\frac{\mathrm{d} s}{\sqrt{s}}\right)$. Then, under $R^{+}$, it holds that

$$
\int_{0}^{\infty} f(s) \mathrm{d} X_{s}=\int_{0}^{\infty} f(s) \mathrm{d} \widehat{X}_{s}+\sqrt{\frac{2}{\pi}} \int_{0}^{\infty} f(s) \frac{\mathrm{d} s}{\sqrt{s}}
$$

where the Wiener integral in the left hand side has been defined in (3.17). 
Proof. It is obvious that the equality (3.29) holds in the case where $f$ is a step function. In the general case, we obtain (3.29) by approximating $f$ by step functions both in $\mathrm{L}^{2}(\mathrm{~d} s)$ and in $\mathrm{L}^{1}\left(\frac{\mathrm{d} s}{\sqrt{s}}\right)$.

Remark 3.13. We cannot dispense with the assumption that $f \in \mathrm{L}^{1}\left(\frac{\mathrm{d} s}{\sqrt{s}}\right)$; in fact, if

$$
f(s)=\frac{1}{\sqrt{s} \log s} 1_{(2, \infty)}(s)
$$

then $f \in \mathrm{L}^{2}(\mathrm{~d} s)$ so that $\int_{0}^{\infty} f(s) \mathrm{d} \widehat{X}_{s}$ exists, while the integral $\int_{0}^{\infty} f(s) \frac{\mathrm{d} s}{\sqrt{s}}$ diverges. See [7] for a very similar discussion.

\section{WIENER INTEGRAL UNDER THE $\sigma$-FINITE MEASURE}

\subsection{A limit theorem for last exit time}

Note that the last exit time from 0 up to time $t$, denoted by $g^{(t)}(X)=\sup \left\{s \leq t: X_{s}=0\right\}$, has, under $W$, the arcsine law:

$$
W\left(g^{(t)}(X) \in \mathrm{d} u\right)=\frac{\mathrm{d} u}{\pi \sqrt{u(t-u)}} \cdot
$$

We need the following limit theorem.

Theorem 4.1. Let $\varphi$ be a non-negative non-increasing function on $(0, \infty)$ such that $\varphi \in \mathrm{L}^{1}\left(\frac{\mathrm{d} u}{\sqrt{u}}\right)$. Then it holds that

$$
\lim _{t \rightarrow \infty} \sqrt{t} \int_{0}^{t} \varphi(u) \frac{\mathrm{d} u}{\sqrt{u(t-u)}}=\int_{0}^{\infty} \varphi(u) \frac{\mathrm{d} u}{\sqrt{u}}
$$

in other words,

$$
\lim _{t \rightarrow \infty} \sqrt{\frac{\pi t}{2}} W\left[\varphi\left(g^{(t)}(X)\right)\right]=\mathscr{W}[\varphi(g(X))]
$$

Remark 4.2. We have a counterexample (see [13], ex. 6.1) if we omit the non-increasingness assumption. Theorem 4.1 is a special case of [13], Lemma 6.3, which plays an important role in penalisation problems.

Proof of Theorem 4.1. It suffices to show that

$$
\lim _{t \rightarrow \infty} \int_{0}^{t} \varphi(u)\left(\sqrt{\frac{t}{t-u}}-1\right) \frac{\mathrm{d} u}{\sqrt{u}}=0 .
$$

Since $\sqrt{a}-\sqrt{b} \leq \sqrt{a-b}$ for $a \geq b \geq 0$, it suffices to show that

$$
\lim _{t \rightarrow \infty} \int_{0}^{t} \varphi(u) \frac{\mathrm{d} u}{\sqrt{t-u}}=0 .
$$

We note that

$$
\int_{t / 4}^{t} \varphi(u) \frac{\mathrm{d} u}{\sqrt{u}} \geq \varphi(t) \int_{t / 4}^{t} \frac{\mathrm{d} u}{\sqrt{u}}=\sqrt{t} \varphi(t) .
$$

Hence it follows from the assumption $\varphi \in \mathrm{L}^{1}\left(\frac{\mathrm{d} u}{\sqrt{u}}\right)$ that $\sqrt{t} \varphi(t) \rightarrow 0$ as $t \rightarrow \infty$. 
Let $0<\varepsilon<1$. First, we have

$$
\begin{aligned}
\int_{\varepsilon t}^{t} \varphi(u) \frac{\mathrm{d} u}{\sqrt{t-u}} & \leq \varphi(\varepsilon t) \int_{\varepsilon t}^{t} \frac{\mathrm{d} u}{\sqrt{t-u}} \\
& =2 \sqrt{\frac{1-\varepsilon}{\varepsilon}}\{\sqrt{\varepsilon t} \varphi(\varepsilon t)\} \underset{t \rightarrow \infty}{\longrightarrow} 0 .
\end{aligned}
$$

Second, we have

$$
\begin{aligned}
\int_{0}^{\varepsilon t} \varphi(u) \frac{\mathrm{d} u}{\sqrt{t-u}} & \leq \int_{0}^{\varepsilon t} \varphi(u) \frac{\mathrm{d} u}{\sqrt{(u / \varepsilon)-u}} \\
& =\frac{1}{\sqrt{(1 / \varepsilon)-1}} \int_{0}^{\varepsilon t} \varphi(u) \frac{\mathrm{d} u}{\sqrt{u}} .
\end{aligned}
$$

Thus we obtain

$$
\limsup _{t \rightarrow \infty} \int_{0}^{t} \varphi(u) \frac{\mathrm{d} u}{\sqrt{t-u}} \leq \frac{1}{\sqrt{(1 / \varepsilon)-1}} \int_{0}^{\infty} \varphi(u) \frac{\mathrm{d} u}{\sqrt{u}} .
$$

Letting $\varepsilon \rightarrow 0+$, we obtain the desired result.

We shall utilize the following lemma.

Lemma 4.3. Let $\varphi$ be a non-negative non-increasing function on $(0, \infty)$ such that $\varphi \in \mathrm{L}^{1}\left(\frac{\mathrm{d} u}{\sqrt{u}}\right)$. Suppose that $\varphi(0+)$ is finite. Then there exist two constants $c_{0}$ and $C_{0}$ such that, for any Borel function $f$, it holds that

$$
c_{0} \int_{0}^{\infty}|f(s)| \frac{\mathrm{d} s}{1+\sqrt{s}} \leq \int_{0}^{\infty} \frac{\mathrm{d} u}{\sqrt{u}} \varphi(u) \int_{0}^{\infty}|f(s+u)| \frac{\mathrm{d} s}{\sqrt{s}} \leq C_{0} \int_{0}^{\infty}|f(s)| \frac{\mathrm{d} s}{1+\sqrt{s}} .
$$

In other words, the norm $\|\cdot\|_{\varphi}$ defined by

$$
\|f\|_{\varphi}=\int_{0}^{\infty} \frac{\mathrm{d} u}{\sqrt{u}} \varphi(u) \int_{0}^{\infty}|f(s+u)| \frac{\mathrm{d} s}{\sqrt{s}}
$$

is equivalent to the norm $\|\cdot\|_{\mathrm{L}^{1}\left(\frac{\mathrm{d} s}{1+\sqrt{s}}\right)}$. In particular, if $f \in \mathrm{L}^{1}\left(\frac{\mathrm{d} s}{1+\sqrt{s}}\right)$, then it holds that

$$
\int_{0}^{\infty}|f(s+u)| \frac{\mathrm{d} s}{\sqrt{s}}<\infty \quad \text { for a.e. } u \in[0, \infty) .
$$

Proof. Changing the order of integration, we have

$$
\begin{aligned}
\|f\|_{\varphi} & =\int_{0}^{\infty} \frac{\mathrm{d} u}{\sqrt{u}} \varphi(u) \int_{u}^{\infty}|f(s)| \frac{\mathrm{d} s}{\sqrt{s-u}} \\
& =\int_{0}^{\infty} \mathrm{d} s|f(s)| \int_{0}^{s} \varphi(u) \frac{\mathrm{d} u}{\sqrt{u(s-u)}} .
\end{aligned}
$$

Applying Theorem 4.1, we see that

$$
\int_{0}^{s} \varphi(u) \frac{\mathrm{d} u}{\sqrt{u(s-u)}} \sim \begin{cases}(1 / \sqrt{s}) \int_{0}^{\infty} \varphi(u) \frac{\mathrm{d} u}{\sqrt{u}} & \text { as } s \rightarrow \infty, \\ \varphi(0+) \pi & \text { as } s \rightarrow 0+.\end{cases}
$$

Thus we obtain the desired result. 


\subsection{Approximation theorems}

Let $\varphi$ be a non-negative non-increasing function on $(0, \infty)$ such that $\varphi \in \mathrm{L}^{1}\left(\frac{\mathrm{d} u}{\sqrt{u}}\right)$ and that $\varphi(0+)$ is finite. We are mainly interested in the measure $\frac{\mathrm{d} u}{\sqrt{2 \pi u}} \Pi^{(u)} \bullet R$, but it will be more convenient to work with the finite measure

$$
\mu_{\varphi}(\mathrm{d} u \times \mathrm{d} X)=\frac{\mathrm{d} u}{C_{\varphi} \sqrt{u}} \varphi(u)\left(\Pi^{(u)} \bullet R\right)(\mathrm{d} X)
$$

where $C_{\varphi}=\int_{0}^{\infty} \varphi(u) \frac{\mathrm{d} u}{\sqrt{u}}$. Note that

$$
\int F(u, X) \mu_{\varphi}(\mathrm{d} u \times \mathrm{d} X)=\frac{1}{\mathscr{W}[\varphi(g(X))]} \int_{\Omega} \mathscr{W}(\mathrm{d} X)[\varphi(g(X)) F(g(X), X)]
$$

for any non-negative measurable function $F$ on $[0, \infty) \times \Omega$. For simplicity, we may choose $\varphi(u)=\mathrm{e}^{-u}$ and write $\mu$ for $\mu_{\varphi}$.

For $f \in \mathcal{S}$ and the coordinate process $X$, we define

$$
J^{(1)}\left(f ; u, X^{(u)}\right)=\int_{0}^{u} f(s) \mathrm{d} X_{s} \quad \text { and } \quad J^{(2)}\left(f ; u, \theta_{u} X\right)=\int_{0}^{\infty} f(s+u) \mathrm{d}\left(\theta_{u} X\right)_{s} .
$$

Proposition 4.4. Let $f \in \mathcal{S}$. Then it holds that

$$
\mu\left[\left|J^{(1)}\left(f ; u, X^{(u)}\right)\right|\right] \leq\|f\|_{\mathrm{L}^{2}(\mathrm{~d} s)}
$$

and that there exists an absolute constant $C_{1}$ such that

$$
\mu\left[\left|J^{(2)}\left(f ; u, \theta_{u} X\right)\right|\right] \leq C_{1}\left\{\|f\|_{\mathrm{L}^{2}(\mathrm{~d} s)}+\|f\|_{\mathrm{L}^{1}\left(\frac{\mathrm{d} s}{1+\sqrt{s}}\right)}\right\} .
$$

Proof. By definition, we have

$$
\mu\left[\left|\int_{0}^{u} f(s) \mathrm{d} X_{s}\right|\right]=\int_{0}^{\infty} \frac{\mathrm{d} u}{\sqrt{\pi u}} \mathrm{e}^{-u}\left\|\int_{0}^{u} f(s) \mathrm{d} X_{s}\right\|_{L^{1}\left(\Pi^{(u)}\right)} .
$$

By the Schwarz inequality and by the Itô isometry (3.11), we have

$$
(4.23) \leq \int_{0}^{\infty} \frac{\mathrm{d} u}{\sqrt{\pi u}} \mathrm{e}^{-u}\left\|\int_{0}^{u} f(s) \mathrm{d} X_{s}\right\|_{\mathrm{L}^{2}\left(\Pi^{(u)}\right)} \leq\left\|\pi_{u} f\right\|_{\mathrm{L}^{2}([0, u], \mathrm{d} s)} \leq\|f\|_{\mathrm{L}^{2}(\mathrm{~d} s)} .
$$

This proves (4.21).

By definition, we have

$$
\mu\left[\left|\int_{0}^{\infty} f(s+u) \mathrm{d} X_{s+u}\right|\right]=\int_{0}^{\infty} \frac{\mathrm{d} u}{\sqrt{\pi u}} \mathrm{e}^{-u} R\left[\left|\int_{0}^{\infty} f(s+u) \mathrm{d} X_{s}\right|\right] .
$$

Using (3.17), we have

$$
\begin{aligned}
R\left[\left|\int_{0}^{\infty} f(s+u) \mathrm{d} X_{s}\right|\right] & \leq R\left[\left|\int_{0}^{\infty} f(s+u) \mathrm{d} B_{s}\right|\right]+\int_{0}^{\infty}|f(s+u)| R\left[\frac{1}{X_{s}}\right] \mathrm{d} s \\
& \leq\|f\|_{\mathrm{L}^{2}(\mathrm{~d} s)}+\sqrt{\frac{2}{\pi}} \int_{0}^{\infty}|f(s+u)| \frac{\mathrm{d} s}{\sqrt{s}}
\end{aligned}
$$


Hence we have

$$
(4.25) \leq\|f\|_{\mathrm{L}^{2}(\mathrm{~d} s)}+\frac{\sqrt{2}}{\pi}\|f\|_{\varphi}
$$

where $\varphi(u)=\mathrm{e}^{-u}$. By Lemma 4.3, we obtain (4.22). The proof is now complete.

Theorem 4.5. Let $f \in \mathrm{L}^{2}(\mathrm{~d} s) \cap \mathrm{L}^{1}\left(\frac{\mathrm{d} s}{1+\sqrt{s}}\right)$. Suppose that a sequence $\left\{f_{n}\right\} \subset \mathcal{S}$ approximates $f$ both in $\mathrm{L}^{2}(\mathrm{~d} s)$ and in $\mathrm{L}^{1}\left(\frac{\mathrm{d} s}{1+\sqrt{s}}\right)$. Then there exist jointly measurable functionals $J^{(1)}\left(f ; u, X^{(u)}\right)$ and $J^{(2)}\left(f ; u, \theta_{u} X\right)$ such that

$$
J^{(1)}\left(f_{n} ; u, X^{(u)}\right) \underset{n \rightarrow \infty}{\longrightarrow} J^{(1)}\left(f ; u, X^{(u)}\right) \quad \text { in } \mu \text {-probability }
$$

and

$$
J^{(2)}\left(f_{n} ; u, \theta_{u} X\right) \underset{n \rightarrow \infty}{\longrightarrow} J^{(2)}\left(f ; u, \theta_{u} X\right) \text { in } \mu \text {-probability }
$$

The limit functionals do not depend on the choice of the approximation sequence. Moreover, it holds for a.e. $u \in[0, \infty)$ and for $\left(\Pi^{(u)} \bullet R\right)(\mathrm{d} X)$-a.e. $X$ that

$$
J^{(1)}\left(f ; u, X^{(u)}\right)=\int_{0}^{u} f(s) \mathrm{d} X_{s} \quad \text { and } \quad J^{(2)}\left(f_{n} ; u, \theta_{u} X\right)=\int_{0}^{\infty} f(s+u) \mathrm{d}\left(\theta_{u} X\right)_{s} .
$$

Proof. This is obvious by Proposition 4.4.

Now we proceed to prove Theorem 1.1 and Theorem 1.3 at the same time.

Proof of Theorem 1.1 and Theorem 1.3. Let $\left\{f_{n}\right\}$ be a sequence of step functions such that $\left\{f_{n}\right\}$ approximates $f$ both in $\mathrm{L}^{2}(\mathrm{~d} s)$ and in $\mathrm{L}^{1}\left(\frac{\mathrm{d} s}{1+\sqrt{s}}\right)$. Since $f_{n}$ is a step function, we have

$$
\int_{0}^{\infty} f_{n}(s) \mathrm{d} X_{s}=I\left(f_{n} ; g(X), X\right)
$$

where $I\left(f_{n} ; u, X\right)=J^{(1)}\left(f_{n} ; u, X^{(u)}\right)+J^{(2)}\left(f_{n} ; u, \theta_{u} X\right)$. Thus Theorem 4.5 shows that

$$
\int_{0}^{\infty} f_{n}(s) \mathrm{d} X_{s} \underset{n \rightarrow \infty}{\longrightarrow} I(f ; g(X), X) \quad \text { in } \mathscr{W}^{G} \text {-probability }
$$

where $I(f ; u, X)=J^{(1)}\left(f ; u, X^{(u)}\right)+J^{(2)}\left(f ; u, \theta_{u} X\right)$ and where $G(X)=\mathrm{e}^{-g(X)} \in \mathrm{L}_{+}^{1}(\mathscr{W})$. By (ii) of Proposition 2.2 , we obtain

$$
\int_{0}^{\infty} f_{n}(s) \mathrm{d} X_{s} \underset{n \rightarrow \infty}{\longrightarrow} I(f ; g(X), X) \quad \text { locally in } \mathscr{W} \text {-measure }
$$

where

$$
I(f ; u, X)=J^{(1)}\left(f ; u, X^{(u)}\right)+J^{(2)}\left(f ; u, \theta_{u} X\right)
$$

This completes the proof. 


\subsection{Continuous modification}

Let us prove Theorem 1.4.

Proof of Theorem 1.4. For the coordinate process $X$ and for $u=g(X)$, we define

$$
{\widehat{\left(\theta_{u} X\right)_{t}}}_{t}=\left(\theta_{u} X\right)_{t}-\sqrt{\frac{2}{\pi}} \int_{0}^{t} \frac{\mathrm{d} s}{\sqrt{s}} \text { for } t \geq 0 \text {. }
$$

Let $f \in \mathrm{L}^{2}([0, T], \mathrm{d} s)$. Then we may define

$$
\left.J^{(3)}\left(f ; u, \theta_{u} X\right)=\int_{0}^{\infty} f(s+u) \mathrm{d} \widehat{\left(\theta_{u} X\right)}\right)_{s} \quad \mu \text {-a.s. }
$$

Applying Theorem 3.11 for $\psi(x)=x^{4}$ and then using the Gaussian property of $\left\{\left(X_{t}\right), W\right\}$, we see that

$$
\mu\left[\left|J^{(3)}\left(f ; u, \theta_{u} X\right)\right|^{4}\right] \leq \int_{0}^{\infty} \frac{\mathrm{d} u}{\sqrt{\pi u}} \mathrm{e}^{-u} R\left[\left|J^{(3)}\left(f ; u, \theta_{u} X\right)\right|^{4}\right] \leq 3\|f\|_{\mathrm{L}^{2}(\mathrm{~d} s)}^{2}
$$

For $t \in[0, T]$, we write $f_{t}=f 1_{[0, t)}$. Set

$$
M(t)=t+\int_{0}^{t}|f(s)|^{2} \mathrm{~d} s
$$

Since the function $v=M(t)$ is continuous and strictly-increasing, there exists its continuous inverse $t=L(v)$. Then, for $0 \leq v_{1}<v_{2} \leq T$, it holds that

$$
\begin{aligned}
& \mu\left[\left|J^{(3)}\left(f_{L\left(v_{2}\right)} ; u, \theta_{u} X\right)-J^{(3)}\left(f_{L\left(v_{1}\right)} ; u, \theta_{u} X\right)\right|^{4}\right] \\
& \leq 3\left(\int_{L\left(v_{1}\right)}^{L\left(v_{2}\right)}|f(s)|^{2} \mathrm{~d} s\right)^{2} \leq 3\left|v_{2}-v_{1}\right|^{2} .
\end{aligned}
$$

From this inequality, we appeal to Kolmogorov's continuity theorem, and we see that there exists a process $\left(K_{v}^{(3)}\left(f ; u, \theta_{u} X\right): v \in[0, M(T)]\right)$ which is a $\mu$-a.s. continuous modification of $\left\{J^{(3)}\left(f_{L(v)} ; u, \theta_{u} X\right): v \in\right.$ $[0, M(T)]\}$. In the same way as above, we may construct a continuous process $\left.\left\{K_{v}^{(1)}\left(f ; u, X^{(u)}\right): v \in[0, M(T)]\right)\right\}$ which is a $\mu$-a.s. continuous modification of $\left\{J^{(1)}\left(f_{L(v)} ; u, X^{(u)}\right): v \in[0, M(T)]\right\}$.

Set

$$
U=\left\{u \in[0, \infty): \int_{0}^{\infty}|f(s+u)| \frac{\mathrm{d} s}{\sqrt{s}}<\infty\right\}
$$

By Lemma 4.3, we see that $U^{c}$ has Lebesgue measure zero. For $u \in U$ and $t \in[0, T]$, we define

$$
I_{t}(f ; u, X)=K_{M(t)}^{(1)}\left(f ; u, X^{(u)}\right)+K_{M(t)}^{(3)}\left(f ; u, \theta_{u} X\right)+\sqrt{\frac{2}{\pi}} \int_{0}^{\infty} f_{t}(s+u) \frac{\mathrm{d} s}{\sqrt{s}}
$$

and, for $u \notin U$ and $t \in[0, T]$, we define $I_{t}(f ; u, X)=0$. Therefore we conclude that the resulting process $\left\{I_{t}(f ; u, X): t \in[0, T]\right\}$ is as desired.

Acknowledgements. The author would like to thank Professors M. Yor and T. Funaki for their fruitful comments. 


\section{REFERENCES}

[1] A. Beck and D.P. Giesy, P-uniform convergence and a vector-valued strong law of large numbers. Trans. Amer. Math. Soc. 147 (1970) 541-559.

[2] T. Funaki, Y. Hariya and M. Yor, Wiener integrals for centered Bessel and related processes, II. ALEA Lat. Am. J. Probab. Math. Stat. 1 (2006) 225-240 (electronic).

[3] T. Funaki, Y. Hariya and M. Yor, Wiener integrals for centered powers of Bessel processes, I. Markov Process. Relat. Fields 13 (2007) 21-56.

[4] T. Funaki, Y. Hariya, F. Hirsch and M. Yor, On the construction of Wiener integrals with respect to certain pseudo-Bessel processes. Stoch. Process. Appl. 116 (2006) 1690-1711.

[5] T. Funaki, Y. Hariya, F. Hirsch and M. Yor, On some Fourier aspects of the construction of certain Wiener integrals. Stoch. Process. Appl. 117 (2007) 1-22.

[6] P. Gosselin and T. Wurzbacher, An Itô type isometry for loops in $\mathbf{R}^{d}$ via the Brownian bridge, in Séminaire de Probabilités XXXI. Lecture Notes in Math. 1655, Springer, Berlin (1997) 225-231.

[7] T. Jeulin and M. Yor, Inégalité de Hardy, semimartingales, et faux-amis, in Séminaire de Probabilités XIII (Univ. Strasbourg, Strasbourg, 1977-1978). Lecture Notes in Math. 721, Springer, Berlin (1979) 332-359.

[8] J. Najnudel, B. Roynette and M. Yor, A remarkable $\sigma$-finite measure on $\mathcal{C}\left(\mathbf{R}_{+}, \mathbf{R}\right)$ related to many Brownian penalisations. C. R. Math. Acad. Sci. Paris 345 (2007) 459-466.

[9] J. Najnudel, B. Roynette and M. Yor, A global view of Brownian penalisations. MSJ Memoirs 19, Mathematical Society of Japan, Tokyo (2009).

[10] B. Roynette and M. Yor, Penalising Brownian paths. Lecture Notes in Math. 1969, Springer, Berlin (2009).

[11] B. Roynette, P. Vallois and M. Yor, Some penalisations of the Wiener measure. Jpn J. Math. 1 (2006) 263-290.

[12] K. Yano, Cameron-Martin formula for the $\sigma$-finite measure unifying Brownian penalisations. J. Funct. Anal. 258 (2010) 3492-3516.

[13] K. Yano, Y. Yano and M. Yor, Penalising symmetric stable Lévy paths. J. Math. Soc. Jpn 61 (2009) 757-798. 